\title{
Análisis de los Mecanismos de Transferencia Tecnológica entre los Sectores Científico-tecnológico y Productivo de Argentina
}

\author{
Ana Inés Malizia', Mabel Sánchez-Barrioluengo², Guillermo Lombera ${ }^{3}$, Elena Castro-Martínez ${ }^{4}$
}

\begin{abstract}
El objetivo de este artículo es analizar el comportamiento de las estructuras de gestión que, en la Argentina, actúan como interfaz en la relación entre los sectores científico-tecnológicos y los productivos. Estas estructuras son denominadas "Oficinas de Vinculación Tecnológica y Transferencia". En ese sentido, se presentan los resultados obtenidos a partir del análisis cuantitativo de los distintos mecanismos de gestión de la interacción entre ambos sectores. A partir del procesamiento estadístico de información de base, se obtuvieron resultados que permitieron definir y categorizar distintas variables relacionadas con las características de estas oficinas y de los sectores de la demanda. El análisis de la relación entre estas variables, permitió obtener información de base a partir de la cual se realizan inferencias acerca del comportamiento general sobre la generación y gestión de los mecanismos que regulan su relación con los sectores científico-tecnológicos y los productivos en la República Argentina.
\end{abstract}

Keywords: mecanismos de transferencia de conocimiento, sistema nacional de innovación, estructuras de interfaz, oficinas de vinculación tecnológica y transferencia

This paper addresses the behavior of technology transfer offices in Argentina, which are defined as interface structures between the productive sector and the scientific, technological and educational areas. In that sense, results from a quantitative analysis of the different interaction mechanisms displayed by these organizations in order to facilitate this interaction are provided. By means of a depth statistical framework it was possible to define and rank different variables related to both the characteristics of these offices and the productive sector. The analysis of the relationship among those variables allowed making inferences about the way technological transferring units behave as facilitators of processes that foster the partnership between those who produce knowledge and those who benefit from its use.

Keywords: knowledge transfer mechanisms, national innovation system, Argentina, interface structures, technology transfer office.

\footnotetext{
'Facultad de Ciencias Exactas y Naturales, Deán Funes3350. E-mail: amalizia@mdp.edu.ar

${ }^{3}$ Facultad de Ingeniería, Juan B. Justo. Consejo Nacional de Investigaciones Científicas y Técnicas. 1,3Universidad Nacional de Mar

del Plata, Juan Bautista Alberdi 2695, 7600 Mar del Plata,Argentina.

${ }^{2,4}$ INGENIO (CSIC-UPV) Universitat Politècnica de València. Camino de Vera s/n, 46022 Valencia, España.
}

ISSN: 07I 8-2724. (http://www.jotmi.org)

Journal of Technology Management \& Innovation (C) Universidad Alberto Hurtado, Facultad de Economía y Negocios. 


\section{Introducción}

El desarrollo de los enfoques de sistemas de innovación (Freeman, 1987; Lundvall, 1992; Nelson, 1993) está íntimamente relacionado con la práctica política. Muchos de estos enfoques centran su atención en la existencia de situaciones donde las relaciones entre diferentes actores del sistema son débiles $y$, al mismo tiempo, donde las divergencias culturales $y$ las prácticas organizativas suponen una barrera a la colaboración y coordinación (Molas-Gallart et al., 2008). En ese sentido, fortalecer la articulación entre el sector científico-tecnológico y el productivo, en tanto que eslabones indispensables de los sistemas de innovación, es un objetivo de importancia superlativa en las condiciones actuales. Frente a las demandas impuestas por la sociedad de innovar productivamente, los avances en el conocimiento generados por los sectores científico-tecnológicos, requieren un nexo para ser aprovechados por los sectores productivos.

Surgen así estructuras de interfaz (Fernández-de-Lucio et al., 1996) que pretenden generar altos niveles de articulación entre ambos sectores. Estas unidades puente actúan como catalizadores de las relaciones entre diferentes actores en el sistema, solventando los problemas que impedían las relaciones entre ellos pero sin estar directamente involucrados en el proceso de innovación en sí mismo (Molas-Gallart et al., 2008). Un ejemplo de estructura de interfaz cuyo fin es la puesta en contacto del mundo académico y empresarial son las oficinas de transferencia de tecnología (TTO), organizaciones que, entre otras funciones, se encargan de potenciar las aplicaciones no académicas generadas desde los resultados de la investigación (Siegel et al., 2003).

Así, el objetivo de este artículo es estudiar el comportamiento de las estructuras de interfaz del entorno científico en el contexto argentino, donde se las conoce como OVTT, a partir de un análisis cuantitativo de los distintos mecanismos de gestión que estas oficinas utilizan para formalizar la vinculación entre los sectores científico-tecnológicos y los productivos. En este estudio, esta relación está definida estrictamente en función de la transferencia de conocimiento obtenido como resultado de investigaciones generadas en el ámbito de distintas instituciones pertenecientes al Sistema Nacional de Ciencia y Tecnología argentino. El artículo se estructura de la siguiente manera: en primer lugar se hace un breve repaso sobre las estructuras de interfaz $y$ su nacimiento en el contexto argentino. Seguidamente se describe la procedencia de los datos utilizados y la metodología de análisis de los mismos. A continuación se detallan los resultados obtenidos $y$, finalmente, se discuten y enuncian las conclusiones que se derivan de ellos así como su posible implicación.

\section{Las estructuras de interfaz}

Entre los diferentes agentes que forman el sistemas de innovación (Freeman, 1987; Lundvall, 1992; Nelson, 1993) se encuentran aquellas organizaciones que actúan como "intermediarias" entre los distintos miembros del propio sistema (Howells, 2006). Estos intermediarios son organizaciones' que actúan de puente (bridging organizations) y buscan establecer y mantener interacciones entre un sistema de actores mientras que al mismo tiempo compensan las debilidades inherentes al propio sistema ${ }^{2}$. En contextos en los que las relaciones son escasas, cobra especial importancia el papel que desempeñan estas estructuras, pues, al reducir las barreras entre los agentes y facilitar el mutuo conocimiento, convierten en efectiva la interacción entre los miembros del sistema dentro de un determinado contexto. Esto se debe a que emergen para asistir a gran cantidad de actores que op-eran en diferentes áreas y que no habían tenido un contacto previo, convirtiéndose así en actores claves para el buen fun-cionamiento del sistema.

Estas organizaciones puente es lo que algunos autores han denominado "estructuras de interfaz" (Fernández-de-Lucio et al., 1996) y se definen como una unidad establecida en un entorno o en su área de influencia, que dinamiza en materia de innovación tecnológica a los elementos de dicho entorno o de otros y fomenta y cataliza las relaciones entre ellos. Pueden pertenecer a uno o más agentes del Sistema de Innovación y encontrarse más o menos próximas a los diferentes entornos del sistema (productivo, tecnológico, financiero, científico). Su objetivo es promover y catalizar las relaciones entre diferentes actores en el sistema, solventando los problemas que impedían las relaciones entre ellos pero sin estar directamente involucrados en el proceso de innovación en sí mismo (Molas-Gallart et al., 2008).

Aunque no todas las estructuras de interfaz citadas tienen los mismos objetivos, pues éstos son variables en función de los agentes entre los cuales realizan su labor intermediadora y el tipo de interacciones o mecanismos que les corresponde gestionar, entre ellas cabe destacar las denominadas Oficinas de Transferencia de Tecnología (TTO), que tienen como responsabilidad la puesta en contacto del mundo académico y el empresarial, de tal forma que potencian las aplicaciones no académicas generadas desde los resultados de la investigación. Además pretenden facilitar la transferencia de estos resultados a otros agentes del sistema de innovación. Por otro lado, también se encargan del asesoramiento de los investigadores en materia de protección industrial e intelectual que son fruto de la colaboración con el sector empresarial y otros agentes externos a la propia institución. Este objetivo nace a partir de la evidencia empírica donde se afirma que la promoción y el fortalecimiento de los vínculos universidad-empresa son esenciales para la creación

ISSN: 07 I8-2724. (http://www.jotmi.org)

Journal of Technology Management \& Innovation @ Universidad Alberto Hurtado, Facultad de Economía y Negocios. 
y desarrollo de capacidades de innovación (Mansfield, 1995, Branscomb et al., 1999, Etzkowitz y Leydesdorff, 2000, Leydesdorff y Meyer, 2007).

Puesto que, en la mayoría de los países, las relaciones ciencia-industria se enmarcan en el ámbito de lo privado, a pesar de ser emprendidas por universidades y otras entidades científicas de carácter público, las Oficinas de Transferencia de Tecnología (OTT) tienen una singularidad: se dedican a la gestión de instrumentos no habituales en el marco de las administraciones públicas, con enfoques opuestos a los que imperan en las entidades públicas. Por ello, sus gestores y su personal técnico presentan perfiles profesionales diversos pero necesitan habilidades tales como la capacidad de negociación, el conocimiento del mercado y de las tecnologías disponibles, aspectos legales y jurídicos de las colaboraciones y de la licencia de derechos de propiedad industrial, etc., no habituales en la función pública. Adicionalmente, la dinámica de estas relaciones, debidas al aprendizaje, ya citado, de los agentes, tiene como consecuencia la necesidad de que los profesionales que desarrollan sus actividades en las OTT adquieran nuevos conocimientos y capacidades de forma constante. La necesidad del aprendizaje continuo, en muchos casos por el intercambio de experiencias, ha dado lugar a la consolidación de redes de ámbito diverso creadas fundamentalmente durante los años 90: la American University Technology Managers (AUTM) creada en 1980 y que agrupa a profesionales de entidades estadounidenses y canadienses; la Association for University Research and Industry Links (AURIL) del Reino Unido a partir de las dos organizaciones que representaron a los gestores de las relaciones con la industria en las universidades desde los años 70; y la Association of European Science \& Technology Transfer Professionals (ASTP) de ámbito europeo. También se han creado diversas redes nacionales de Oficinas de Transferencia de Tecnología como la Red de Oficinas de Transferencia de Resultados de Investigación (OTRI) en España; la Réseau de Coopération des services Universitaires de Relations Industrielles et Economiques (CURIE) en Francia; la Network per la Valorizzazione della ricerca universitaria en Italia. Siguiendo el ejemplo europeo de la red PROTON Europe (que agrupa a 116 OTT y las anteriores redes nacionales), en el ámbito latinoamericano, en 2004 se dio un nuevo impulso a estas actividades al crearse la Asociación de Unidades de Vinculación de las Universidades argentinas.

\section{I. Breve reseña del contexto de las relaciones cien- cia-empresa en Argentina y de sus estructuras de interfaz}

Si bien en la República Argentina existe un cierto grado de consolidación en la relación ciencia-empresa, sus orígenes no van más allá de los últimos treinta años y está marcada fuertemente por los vaivenes de las numerosas crisis económicas que históricamente ha atravesado el país. Existe una particular situación dada por la gran concentración geográfica del sector productivo, ya que de las 23 provincias que conforman el territorio argentino en sólo tres de ellas (Buenos Aires, Santa Fe y Córdoba) se genera el 68\% del total de la producción nacional. Paralelamente, se registra una similar situación en la distribución geográfica de los recursos humanos relacionados con el sector científico-tecnológico nacional. A partir de datos aportados por la Secretaría de Políticas Universitarias (SPU) del Ministerio de Educación, el $80 \%$ de los investigadores formados se concentran en las regiones Metropolitana, Bonaerense, Centro Este y Centro Oeste, que se corresponden con las tres provincias anteriormente mencionadas.

La incipiente difusión de las nuevas tecnologías en los sectores productivos argentinos está teniendo impactos muy fuertes sobre la organización de la producción, verificándose fuertes incrementos de la productividad, así como cambios significativos en las relaciones obrero-empresariales (Kosacoff, 1993). Uno de los primeros grandes desafíos es el de generar condiciones para aprovechar las experiencias positivas que involucran considerables acervos tecnológicos, económicos y de calificación de los recursos humanos, teniendo como punto de partida un marco de estabilidad micro y macroeconómica. En este proceso, la generación y lo incorporación de progreso técnico se convierte en un aspecto clave del dinamismo productivo. La posibilidad de acceder a niveles crecientes de competitividad y mantenerlos en el largo plazo no puede circunscribirse a la acción de un agente económico individual (Kosacoff, 1993). Este autor señala que, la "noción sistémica" de competitividad reemplaza a los esfuerzos individuales que, si bien son condición necesaria para lograr este objetivo, deben estar acompañados, necesariamente, por innumerables aspectos que conforman el entorno de las firmas (desde la infraestructura física, el aparato científico tecnológico, la red de proveedores y subcontratistas, los sistemas de distribución y comercialización hasta los valores culturales, las instituciones, el marco jurídico, etc.).

Uno de los hitos formales del inicio de las actividades de vinculación entre los sectores científicos y los productivos en la Argentina fue la creación, en 1984, de una oficina de transferencia tecnológica en el seno del Consejo Nacional de Investigaciones Científicas y Técnicas (CONICET), dependiente del entonces Ministerio de Educación y Cultura de la Nación. Posteriormente, la promulgación de la Ley 23.877 de Promoción y Fomento de la Innovación Tecnológica en 1992, posibilitó la asignación de fondos públicos concursables, destinados a financiar proyectos tecnológicos innovadores. Este hecho dio lugar a la creación de las Unidades de Vinculación Tecnológica (UVT) de carácter público y privado, dedicadas a la gestión de la transferencia de tecnología y de 
la vinculación con sectores de la industria. A partir del año 1994, la Secretaria de Políticas Universitarias (dependiente del Ministerio de Educación de la Nación Argentina) inicia acciones para desarrollar estas áreas en las Universidades Argentinas. Por otra parte, la promulgación de una nueva Ley Nacional de Educación Superior en el año 1995, habilita institucionalmente a las Universidades Nacionales (UUNN) a constituirse por naturaleza como Unidades deVinculación Tecnológica. Estas comienzan a formalizar masivamente la creación de oficinas dedicadas a la gestión de las actividades de vinculación tecnológica con el sector productivo que hasta ese momento, con algunas excepciones, eran incipientes. Por otra parte, en al año 2004, el Consejo Universitario Nacional (CIN), integrado por los rectores de todas las UUNN de gestión pública gratuita, crea la Red Nacional de Vinculación Tecnológica (REdVITEC). En la actualidad, esta red representa la más fuerte apuesta del sistema universitario púbico argentino a la coordinación, desarrollo y difusión conjuntos de sus acciones en materia de vinculación tecnológica con los sectores productivos y sociales del país. El conjunto de las acciones políticas e institucionales anteriormente mencionadas, ha llevado a que en la actualidad exista una gran diversidad de unidades de vinculación tecnológica de carácter público (dependientes de Universidades Nacionales y organismos nacionales y provinciales de ciencia y tecnología) y privado (dependientes de universidades privadas, empresas, parques tecnológicos, fundaciones, asociaciones civiles y cooperativas). Cabe aclarar que las Universidades Nacionales de gestión pública son gratuitas y financiadas exclusivamente por el Estado Nacional. A los efectos de este trabajo, este universo de estructuras de interfaz se ha unificado bajo la denominación genérica de "Oficinas de Vinculación Tecnológica y Transferencia” (OVTT).

\section{Materiales y Métodos}

\section{I. Datos utilizados}

La información de base utilizada para el desarrollo de este estudio procede de un informe ${ }^{3}$ cuya finalidad es diagnosticar las capacidades institucionales de las OVTT argentinas en el quinquenio 2004-2009 y, así, abrir el debate sobre su funcionamiento y situación institucional en ese momento. Los datos fueron recogidos durante 2010 a partir de la "Encuesta diagnóstica institucional" que se compone de 34 preguntas dirigidas a las máximas autoridades de las oficinas (directores, gerentes, etc.). Este estudio se basa en las preguntas las correspondientes a los servicios prestados por las citadas OVTT y las que permiten caracterizar las oficinas y sus demandantes. Las OVTT, de acuerdo a su distribución geográfica, fueron asignadas a distintas regiones: Metropolitana, Bonaerense, Centro Este, Centro Oeste, Noreste y Sur, de acuerdo a la división territorial realizada por el Ministerio de Educación. Del universo de 300 OVTT que actualmente están registradas en el marco del Sistema Nacional de Ciencia y Tecnología, se seleccionó una muestra de $100^{4}$, según el criterio de selección aplicable en este caso de mantener la proporcionalidad de OVTT por región. Se recibieron 7I encuestas válidas, lo que supone una tasa de respuesta del $71 \%$, y da lugar a la muestra en la que se basa este estudio.

\subsection{Variables e Hipótesis}

\section{Variables dependientes}

Como se mencionó anteriormente, el objetivo del estudio consiste en comprobar cuál es el comportamiento de las OVTT argentinas a partir del análisis de los distintos mecanismos utilizados por estas oficinas para gestionar formalmente la relación entre el sector científico-tecnológico y el productivo. Estos mecanismos se centran en acuerdos de carácter contractual que pueden ser de diferente índole: cambio de escala, investigación concertada, servicios técnicos, consultorías, desarrollos, capacitación de recursos humanos o de licencias.

A los efectos de este estudio, se reagruparon las diferentes modalidades de los acuerdos contractuales definidos en la encuesta original y se tipificaron utilizando la clasificación de indicadores de mecanismos de interacción propuestos por Molas-Gallart et al. (2002). Si bien conceptualmente estos indicadores elaborados fueron originalmente concebidos para evaluar la denominada "Tercera Misión" de las universidades, pueden extrapolarse al caso de las entidades de interfaz $y$, en concreto, al de las OVTT argentinas puesto que son intermediarias entre los sectores científicotecnológico y el sector productivo. La clasificación de los mecanismos de gestión ${ }^{5}$ resultante de esta reorganización se presenta en la Tabla I. (Ver sig. pag)

Estos cuatro mecanismos de interacción (contratos de I+D, asesoramiento, cursos de formación y comercialización de resultados) son gestionados, en mayor o menor medida, por las OVTT argentinas. La clasificación de los contratos en estos grupos nos permitirá posteriormente distinguir entre la variedad y cantidad de mecanismos que cada OVTT lleva a cabo. Para ello se utilizan tanto variables dicotómicas, que miden si las OVTT realizan o no este tipo de contratación, como el número de contratos en cada categoría. A partir de esa información, se han creado las variables "Diversidad" y "Cantidad" como identificadoras de los mecanismos de interacción. Para la creación de dichas variables se ha seguido la metodología propuesta por D'Este y Patel (2007) ${ }^{6}$. En ese trabajo, los autores conjugan en esas dos únicas variables las tres propiedades que deben reunir aquellas que miden la variedad de mecanismos de interacción: (a) ¿Cuántas categorías tenemos?; (b) ¿Cuántos de cada tipo tenemos?; (c) ¿Cómo 
de diferentes son las categorías del resto? Estas propiedades fueron propuestas por Stirling (1998). Por un lado, la variable Diversidad estima cuantos mecanismos de interacción diferentes están incluidos entre los servicios de gestión que presta una determinada OVTT. En nuestro caso la variable toma valores entre 0 y 4 . Así, si la OVTT no gestiona ninguno de los mecanismos de interacción antes definidos, esta variable tomará valor 0 ; si gestiona uno de ellos, tomará valor I, y así sucesivamente hasta incluir aquellas OVTT que gestionan toda la variedad de mecanismos de interacción definidos en cuyo caso, la variable Diversidad tomará su máximo valor, 4. Por otra parte, la variable Cantidad estima el número de contratos que corresponden a los distintos mecanismos de interacción gestionados por las OVTT más frecuentemente que la media. Esta variable proporciona información sobre la frecuencia de cada mecanismo de interacción. Para cada uno de ellos, se calcula la media de acuerdos contractuales gestionados por la totalidad de OVTT. Luego se genera una variable dicotómica que toma valores entre 0 y 4 . Estos valores indican en cuántos de los mecanismos de interacción definidos, las distintas OVTT están por encima de la media de contratos calculada. El valor I indica que una determinada OVTT está por encima de la media global en alguno de los mecanismos de interacción definidos. Así sucesivamente hasta incluir todas aquellas OVTT que gestionan contratos por encima de la media en toda la variedad de mecanismos de interacción, en cuyo caso la variable Cantidad tomará su máximo valor (4).

\section{Variables independientes}

La manera en que una OVTT actúa se ve influenciada por las características de la propia oficina así como por los agentes con los que se relaciona y demandan sus servicios. Por ello, las variables que se utilizan como variables independientes en este estudio se dividen en dos grupos: características de la OVTT y características del agente demandante. En el Anexo I se muestran los estadísticos descriptivos de todas las variables del estudio.
En cuanto a las características de la OVTT se tienen en cuenta su experiencia, número de empleados, porcentaje de doctores que forman parte de su personal, ingresos económicos, ámbito de pertenecía, y región geográfica a la que pertenece. El hecho de que estas oficinas estén distribuidas a lo largo del territorio argentino supone importantes diferencias en cuanto al tejido productivo existente a su alrededor. Puesto que la disposición territorial de las OVTT puede influir en las relaciones que establecen con el sector productivo, las oficinas han sido agrupadas en siete regiones geográficas: Buenos Aires, Centro Este, Centro Oeste, Metropolitana, Noroeste, Noreste y Sur. Esta variable se utilizará como control en el análisis de los datos. Es importante tener en cuenta la región, porque aquellos investigadores situados en regiones con un alto nivel de inversión en nuevo conocimiento pueden más fácilmente acceder y generar nuevas ideas científicas. Es decir, el conocimiento tiende a extenderse dentro de las regiones geográficamente delimitadas (Glaeser et al., 1992;Audretsch y Feldman, 1996).

Para el resto de variables independientes definidas se plantean las siguientes hipótesis. (a) Experiencia: medimos la experiencia a través del número de años transcurridos desde la creación de la OVTT. Esta variable pretende medir el posible efecto learning by doing en sus outputs, de tal forma que las estructuras de interfaz más antiguas se benefician de su experiencia en comparación con las más jóvenes y se genera un impacto positivo en su eficiencia (Curi et al., 20I2). En este sentido, la hipótesis puesta a prueba es que a mayor experiencia de la OVTT mayor será la diversidad y cantidad de mecanismos de interacción gestionados. (b) Empleados: Número de personas con las que cuenta la OVTT (tanto dedicadas a tareas profesionales como administrativas). En relación al personal de la OVTT se plantea la hipótesis de que a mayor número de empleados, mayor será la diversidad y cantidad de mecanismos gestionados. Esta hipótesis está en la línea de los resultados obtenidos por otros autores (Siegel et al., 2003), que concluyen que un incremento en el personal de estas estructuras de in-

\begin{tabular}{l|l}
\multicolumn{1}{c|}{ Mecanismos de interacción } & \multicolumn{1}{c}{ Tipo de contratos que incluyen } \\
\hline Contratos de Investigación (I+D) & $\begin{array}{l}\text { Cambio de escala } \\
\text { Investigación Concertada }\end{array}$ \\
\hline \multirow{2}{*}{ Asesoramiento } & $\begin{array}{l}\text { Técnicos } \\
\text { Consultoría } \\
\text { Desarrollo }\end{array}$ \\
\hline Cursos y Actividades de Formación & Capacitación de RRHH \\
\hline Comercialización de resultados & $\begin{array}{l}\text { De licencias } \\
\text { De cesión de titularidad } \\
\text { Acuerdos de transferencia }\end{array}$ \\
\hline
\end{tabular}

Tabla I. Mecanismos de interacción utilizados por las OVTT para relacionarse con el entorno. Nota:Adaptado de Molas-Gallart et al. (2002).

ISSN: 07 I8-2724. (http://www.jotmi.org)

Journal of Technology Management \& Innovation @ Universidad Alberto Hurtado, Facultad de Economía y Negocios. 
terfaz incrementa el número de convenios, pero no los ingresos adicionales. (c) Doctores: para tener en cuenta el nivel educativo del personal de la OVTT, se ha calculado el porcentaje del personal que ha adquirido el grado de doctor respecto del total de empleados. El nivel educativo y la relación entre pares facilita la relación entre los investigadores $y$ el personal de la OVTT $y$, por tanto, nuestra hipótesis es que esto influirá positivamente en la variedad y diversidad de mecanismos de interacción. (d) Ingresos: esta variable refleja el porcentaje de ingresos de la OVTT que son gestionados por ella misma. En relación a los recursos disponibles, Siegel et al. (2003) concluyen para el caso de las oficinas de transferencia de EE.UU. que estas estructuras de interfaz son más eficientes cuando se implementa una estrategia de maximización de ingresos. Teniendo esto en cuenta, planteamos la hipótesis de que a mayor capacidad de autofinanciación de la OVTT, menor será la diversidad de mecanismos y menor la cantidad gestionada. (e) Tipo: refleja la condición pública o privada de la OVTT.Variable dicotómica que toma el valor I si la OVTT pertenece al sector público y 0 si pertenece al sector privado. Esta diferencia es una cuestión específica de las OVTT argentinas, donde el tipo de estructura influye y ejerce diferencias básicas entre ambos tipos en lo que respecta a la financiación. Esta variable también fue utilizada como control.

Respecto a las variables independientes relacionadas con el demandante de la gestión de servicios, se definieron las siguientes: (a) Sector demandante: permite recoger la variedad de sectores de la demanda de la que provienen los clientes de una determinada OVTT. Para la creación de esta variable se realizó un paso previo donde se han creado 4 variables dummy que toman el valor I si la OVTT tiene demandantes de un sector concreto (primario, industria, construcción, servicios) y 0 en caso de no tener demandantes de ese sector. Puesto que las OVTT pueden tener demandantes de varios sectores al mismo tiempo, se calculó el número de sectores diferentes que demandan servicios de la OVTT, de tal forma que se sumaron las 4 variables dummies anteriores. Esta nueva variable permite comprobar si una mayor diversidad de demandantes influye en la cantidad y diversidad de mecanismos de transferencia ${ }^{7}$. (b) Tipo demandante: permite comprobar si tener más de un sector como demandante de servicio influye en cada una de las variables dependientes definidas. Variable dicotómica que toma el valor I si la OVTT ha prestado servicios mayoritariamente a empresas externas (públicas o privadas) y 0 si en su mayoría lo ha hecho a la comunidad científica. Se espera que cuanto mayor sea el número de sectores que demandan actividades, mayor sea la variedad y cantidad de mecanismos de interacción, puesto que las necesidades de los sectores varían debido a sus características intrínsecas. (c) Distancia geográfica: permite evaluar la influencia del ámbito geográfico de las entidades demandantes. Variable dicotómica que toma el valor I si la OVTT ha prestado servicios mayoritariamente a entidades en su ámbito local o provincial y 0 si lo ha prestado en mayor medida a empresas de ámbito regional, nacional o internacional. En este caso, la proximidad geográfica, entendida como distancia física y no cultural, es un factor importante a tener en cuenta para la transferencia de resultados de investigación, puesto que la proximidad geográfica a las universidades permite el acceso al conocimiento que en ella se genera (Audretsch et al., 2005).

\subsection{Análisis de los datos}

Se realizaron dos tipos de análisis. En primer lugar, se utilizaron métodos estadísticos para describir los mecanismos de interacción de las OVTT. En segundo lugar, se realizó una regresión logística ordinal para analizar la influencia de las variables independientes en la cantidad y diversidad de los mecanismos de interacción. La elección de este tipo de regresión se debe a que las variables dependientes son categóricas ordinales (Hair et al., 1998).

\section{Resultados}

\section{I.Análisis de los mecanismos de interacción}

En la Tabla 2 se presenta el análisis de los mecanismos de interacción para analizar su relación con el sector de origen y ubicación geográfica de las distintas OVTT. Es importante resaltar del análisis de porcentajes obtenidos que los contratos de asesoramiento son las herramientas más fuertes de interacción con el entorno utilizadas por las OVTT independientemente del tipo, seguidos por los de formación de $\mathrm{RRHH}$ y de gestión de I+D. Al analizar la variable definida como Comercialización se verifica que este mecanismo de interacción resulta ser el menos habitual en las OVTT. Sin embargo, no se verificaron diferencias significativas entre los mecanismos de interacción utilizados por las OVTT distinguiendo entre públicas y privadas ni atendiendo a la región geográfica considerada $(P>0.05)$. Por otro lado, atendiendo al número de contratos que se gestionan, los datos indican que las OVTT de tipo privado son las que gestionaron, en promedio, un mayor número de acuerdos de cada una de las modalidades contractuales.

El análisis de estas mismas variables en función de la región geográfica de origen de las OVTT, indica que las pertenecientes a las regiones Centro Oeste, Metropolitana y Sur son las que mayoritariamente gestionan contratos de asesoramiento. Por otra parte, las OVTT de las regiones Centro son las que han gestionado la mayor proporción de actividades de gestión de I+D en comparación con las restantes. Respecto a las actividades de formación, salvo la Región Metropolitana y Noreste, las OVTT de las restantes regiones gestionan este mecanismo de interacción en porcentajes 
elevados. Un dato notable es que la actividad de comercialización resulta ser el mecanismo menos desarrollado por la totalidad de las OVTT relevadas, ya que representa menos de un $25 \%$ respecto a los restantes mecanismos de gestión de la interacción utilizados.

En segundo lugar se analiza los mecanismos de transferencia de las OVTT en relación a las características del demandante de servicios (Tabla 3). En este caso se han tenido en cuenta las variables dummies que hacen referencia a los cuatro sectores empresariales con el fin de aportar unos resultados más interesantes. Claramente los resultados indican que, independientemente del sector demandante de la actividad, el mecanismo de gestión más solicitado es el de asesoramiento. Las diferencias aparecen en el mecanismo que ocupa la segunda posición.Así, mientras que existen más OVTT que gestionan contratos de I+D con empresas de los sectores primario, industrial y servicios, un mayor porcentaje de estas oficinas realizan contratos de formación con el sector de la construcción (83,3\%). Esto indica que el sector de la construcción está demandando unos servicios diferentes al resto de sectores. En referencia al análisis de la relación entre el número de mecanismos de interacción gestionados por las OVTT y los sectores de la demanda que los solicitan puede observarse que el sector primario requiere a las OVTT fundamentalmente la gestión de contratos de asesoramiento y formación. Por otra parte, existen diferencias estadísticamente significativas $(P<0.05)$ entre las OVTT que interaccionan con empresas del sector primario. En ese sentido, la interacción con el sector primario impulsa a que las primeras gestionan un número mayor de contratos de I+D y asesoramiento. Respecto al sector industrial, este es demandante en mayor medida de la gestión de contratos de asesoramiento seguidos por los de I+D. Las demandas del sector de la construcción son mucho mayores en asesoramiento y comercialización.

Por otra parte, si se tiene en cuenta el tipo de demandante (Comunidad científica versus Clientes externos), se observa que el $84,4 \%$ de las OVTT realizan indistintamente servicios de asesoramiento y de formación a clientes externos (públicos y/o privados). Por otra parte, la comunidad científica requiere fundamentalmente apoyo en la gestión de mecanismos de asesoramiento $(71,1 \%)$. Además, los sectores demandantes que se encuentran próximos al ámbito geográfico de la OVTT (misma localidad o provincia) solicitan fundamentalmente $(74,5 \%)$ la gestión de servicios de asesoramiento, mientras que los sectores de la demanda alejados del área de influencia de la respectiva OVTT, solicita además la gestión de I+D (80\%).

\subsection{Relaciones entre la Diversidad y Cantidad de mecanismos de interacción}

En esta sección se analizan los factores que influyen en la diversidad y cantidad de mecanismos de interacción gestionados por las distintas OVTT. La Tabla 4 aporta un análisis de las frecuencias de estas dos variables creadas siguiendo la metodología de D'Este y Patel (2007). Así, mientras que el $8,5 \%$ de las OVTT declaró no gestionar ninguno de los mecanismos de interacción definidos, el 38\% informó gestionar tres tipos diferentes. Por otra parte, en lo referente al número de contratos gestionados (cantidad), el $40 \%$ de las OVTT están por encima de la media en tres de los cuatro mecanismos de interacción y, sólo el 3,6\% está por debajo de esta media en todos ellos.

\begin{tabular}{|llc|c|c|c|} 
& & I+D & $\begin{array}{c}\text { Asesora- } \\
\text { miento }\end{array}$ & $\begin{array}{c}\text { For- } \\
\text { mación }\end{array}$ & $\begin{array}{c}\text { Comercial- } \\
\text { ización }\end{array}$ \\
\hline \multirow{2}{*}{ Tipo } & Pública & 68,3 & 75,6 & 70,7 & 22,0 \\
& Privada & 70,0 & 76,7 & 60,0 & 23,3 \\
& R. Buenos Aires & 66,7 & 66,7 & 77,8 & 44,4 \\
& R. Centro Este & 80,0 & 73,3 & 60,0 & 13,3 \\
Región & R. Centro Oeste & 80,0 & 90,0 & 70,0 & 30,0 \\
& R. Metropolitana & 54,5 & 90,9 & 54,5 & 18,2 \\
& R. Noreste & 60,0 & 40,0 & 40,0 & 20,0 \\
& R. Noroeste & 77,8 & 66,7 & 77,8 & 33,3 \\
Total & R. Sur & 58,3 & 83,3 & 75,0 & 8,3 \\
\hline
\end{tabular}

Tabla 2. Mecanismos de interacción según la estructura organizativa y ubicación geográfica de la OVTT.

Nota: No existen diferencias estadísticamente significativas entre el tipo de OVTT ni la región en la que se sitúan en ninguna de las modalidades de interacción (test chi-cuadrado). Los porcentajes no suman 100\% porque una OVTT puede haber contestado I,2,3 o 4 mecanismos de interacción.

ISSN: 07 I8-2724. (http://www.jotmi.org)

Journal of Technology Management \& Innovation (c) Universidad Alberto Hurtado, Facultad de Economía y Negocios. 
Para evitar posibles sesgos en los resultados debido a la naturaleza de las variables independientes (empleados está sesgada a la izquierda, ya que la mayoría de las OVTT presenta un bajo número de empleados), se utilizó en el modelo de regresión el logaritmo neperiano de esta variable. Por otro lado, en el anexo II b se muestra la matriz de correlaciones para las variables independientes. Las bajas correlaciones entre estas variables son síntoma de una ausencia de problemas de multicolinealidad.Además, se muestran los índices de tolerancia para las variables independientes que, puesto que todos los valores son mucho mayores que 0,2 , son síntoma de que los problemas de multicolinealidad no están afectando a nuestro modelo de regresión.

La Tabla 5 incluye los resultados del modelo de regresión. Las columnas (I) y (2) analizan la influencia de las variables independientes en la diversidad de mecanismos de interacción, y las variables (3) y (4) su relación sobre la cantidad. En ambos casos, las columnas impares sólo incluyen las variables relacionadas con las características de la OVTT y las columnas pares, además de las anteriores, las características del demandante del servicio. Los valores del Akaike Information Criterion (AIC) y el Bayesian Information Criterion (BIC) son medidas de la bondad del ajuste de los modelos propuestos. En el caso de la diversidad, la introducción de nuevas variables en el modelo 2 mejora los resultados del modelo I al obtener un valor más bajo para estos dos indicadores. En cambio, en el caso de la cantidad, la introducción de nuevas variables no mejora el modelo original, puesto que no se muestra significación para ninguna de las nuevas variables del modelo 4 .

Así, cuando sólo se consideran las características de la OVTT, se confirma que la experiencia y número de empleados influyen positivamente en la diversidad de mecanismos de interacción gestionados. Estos resultados fueron estadísticamente significativos. Por una parte, por cada año transcurrido desde el inicio de las actividades de la OVTT, la diversidad de mecanismos aumenta en un $7,8 \%$. Por la otra, por cada empleado extra en la OVTT, se incrementan en casi dos unidades $(1,77)$ los mecanismos sobre los que se ofrecen servicios. La relación entre el porcentaje de doctores en

\begin{tabular}{|c|c|c|c|c|c|}
\hline & & $\mathbf{I}+\mathbf{D}$ & $\begin{array}{c}\text { Ase- } \\
\text { sora- } \\
\text { miento }\end{array}$ & $\begin{array}{c}\text { For- } \\
\text { mación }\end{array}$ & $\begin{array}{c}\text { Comer- } \\
\text { cial- } \\
\text { ización }\end{array}$ \\
\hline \multicolumn{2}{|l|}{ Sector Primario } & 73,5 & 79,6 & 69,4 & 28,6 \\
\hline \multicolumn{2}{|l|}{ Sector Industrial } & 72,2 & 79,6 & 68,5 & 25,9 \\
\hline \multicolumn{2}{|l|}{ Sector Construcción ${ }^{\mathrm{b}, \mathrm{c}}$} & 72,2 & 88,9 & 83,3 & 44,4 \\
\hline \multicolumn{2}{|l|}{ Sector Servicios ${ }^{\mathrm{a}}$} & 71,7 & 81,7 & 66,7 & 23,3 \\
\hline \multirow{2}{*}{ Tipo demandante ${ }^{b}$} & Com. Científica & 63,2 & 71,1 & 52,6 & 21,1 \\
\hline & Clientes Externos & 78,1 & 84,4 & 84,4 & 25,0 \\
\hline \multirow{2}{*}{ Proximidad Geográfica } & Local/Provincial & 64,7 & 74,5 & 66,7 & 21,6 \\
\hline & Regional/Nacional/Ext & 80,0 & 80,0 & 65,0 & 25,0 \\
\hline \multicolumn{2}{|l|}{ Total } & 69,0 & 76,1 & 66,2 & 22,5 \\
\hline
\end{tabular}

Tabla 3. Mecanismos de interacción según las características del demandante.

Existen diferencias estadísticamente significativas entre la variable y la gestión de contratos de asesoramiento al nivel $5 \%$. b Existen diferencias estadísticamente significativas entre la variable y la gestión de contratos de formación al nivel 5\%.c Existen diferencias estadísticamente significativas entre la variable y la gestión de contratos de asesoramiento al nivel $5 \%$. Los porcentajes no suman $100 \%$ porque una

OVTT puede haber contestado I,2,3 o 4 mecanismos de interacción.

\begin{tabular}{|ccc|} 
& Diversidad & Cantidad \\
\hline $\mathbf{0}$ & $8,5 \%$ & $3,6 \%$ \\
$\mathbf{1}$ & $18,3 \%$ & $18,2 \%$ \\
$\mathbf{2}$ & $19,7 \%$ & $20,0 \%$ \\
$\mathbf{3}$ & $38,0 \%$ & $40,0 \%$ \\
$\mathbf{4}$ & $15,5 \%$ & $18,2 \%$ \\
\hline Total & $100 \%$ & $100 \%$ \\
$\mathbf{N}^{\mathbf{o}}$ obs. & 71 & 55 \\
\hline
\end{tabular}

Tabla 4. Diversidad y Cantidad de mecanismos de interacción

ISSN: 07 I8-2724. (http://www.jotmi.org)

Journal of Technology Management \& Innovation @ Universidad Alberto Hurtado, Facultad de Economía y Negocios. 
la OVTT y la diversidad de mecanismos gestionados no fue estadísticamente significativa. Cuando se incluyen las características del demandante, se producen algunas diferencias con los resultados anteriores. La experiencia continúa siendo una variable que influye en la diversidad de mecanismos $y$, sin embargo, el tamaño de la oficina expresado en el número de empleados, deja de ser una variable de influencia. Por otra parte, ciertas características del demandante adquieren relevancia estadística. En este sentido, cuanto mayor es la diversidad de los sectores de la demanda, mayor será la diversidad de mecanismos de interacción gestionados por las OVTT. Aunque obvio, los resultados indican que la diversidad de mecanismos que gestionan las OVTT es significativamente mayor cuanto mayor es el número de clientes externos (públicos o privados).

Al analizar la variable que mide la cantidad de contratos gestionados, se observa que el tamaño de la OVTT es la única variable que está significativamente asociada con el aumento de la cantidad de mecanismos de interacción gestionados. Así, cuanto mayor es el tamaño de la OVTT mayor es el número de categorías en las que las OVTT están por encima de la media. Es importante resaltar que, al igual que ocurría con la diversidad, el hecho de contar con personal con grado académico de doctor, si bien el valor es negativo, no resulta ser estadísticamente significativo en el aumento de la frecuencia de contratos gestionados por la OVTT. Finalmente, cuando incluimos en el modelo de regresión las variables referentes a las características del demandante en su conjunto para la cantidad de mecanismos (columna 4), no encontramos asociación estadísticamente significativa entre ninguno de los factores estudiados.

\section{Discusión}

A continuación se discutirán brevemente aquellos resultados que se consideran de mayor importancia y consistencia para caracterizar el desempeño de las OVTT durante el período de estudio. En relación a la diversidad de mecanismos de interacción utilizados, las OVTT públicas y privadas en los distintos ámbitos geográficos, no se diferenciaron entre sí. Sin embargo, puede destacarse que la actividad de asesoramiento fue la más ampliamente gestionada por la totalidad de OVTT. Esto resultado es comprensible si se considera que esta es la actividad de menor complejidad y más ágilmente gestionable. Por otra parte, la formación de recursos humanos y de gestión de I+D son las actividades que siguen en orden de importancia para las OVTT públicas, mientras que para las privadas el orden se invierte. Si consideramos que la mayoría de las OVTT públicas están bajo la órbita de universidades y que la formación de RRHH e I+D son las actividades naturales de estas instituciones, el resultado es el esperado. La comercialización es la actividad menos gestionada por todas las OVTT. Sólo es demandada por el sector productivo en un $25 \%$ respecto a las restantes. Esto probablemente se relacione con que los profesores universitarios

\begin{tabular}{|c|c|c|c|c|}
\hline & \multicolumn{2}{|c|}{ Diversidad } & \multicolumn{2}{|c|}{ Cantidad } \\
\hline & (1) & (2) & (3) & (4) \\
\hline \multicolumn{5}{|l|}{ Características OVTT } \\
\hline Experiencia & $0,078 * *(0,035)$ & $0,063 *(0,037)$ & $0,039(0,038)$ & $0,031(0,040)$ \\
\hline Empleados (ln) & $0,575^{*}(0,314)$ & $0,585(0,366)$ & $0,708 *(0,379)$ & $0,538(0,411)$ \\
\hline Doctores & $-0,026(0,021)$ & $-0,01(0,022)$ & $-0,012(0,026)$ & $0,001(0,029)$ \\
\hline Ingresos & $0,009(0,007)$ & $0,008(0,007)$ & $-0,012(0,009)$ & $-0,013(0,010)$ \\
\hline Tipo & $0,393(0,592)$ & $0,438(0,600)$ & $0,310(0,751)$ & $0,359(0,760)$ \\
\hline \multicolumn{5}{|l|}{ Características Demandante } \\
\hline Sector & & $0,586 * *(0,275)$ & & $0,236(0,370)$ \\
\hline Demandante & & $1,219 * *(0,507)$ & & $0,491(0,671)$ \\
\hline Ámbito geográfico & & $0,277(0,565)$ & & $-0,545(0,756)$ \\
\hline Variable de control: región & Sí & Sí & Sí & Sí \\
\hline $\mathbf{N}^{0}$ observaciones & 71 & 70 & 55 & 55 \\
\hline AIC & 223,9 & 214,6 & 147,4 & 151,6 \\
\hline BIC & 257,8 & 255 & 177,5 & 187,7 \\
\hline
\end{tabular}

Tabla 5. Factores que influyen en la Diversidad y Cantidad de los mecanismos de interacción.

$* \mathrm{P}<0, \mathrm{I}$; **P $<0,05$. Se toma como categorías de referencia los valores más pequeños (valor o) de cada una de las variables Categoría de referencia para la variable de control: Región Buenos Aires.

ISSN: 07 I8-2724. (http://www.jotmi.org)

Journal of Technology Management \& Innovation @ Universidad Alberto Hurtado, Facultad de Economía y Negocios. 
tienen poca experiencia en la protección industrial de los resultados ya que es una actividad que aún no ha entrado a formar parte del quehacer de la comunidad científica que investiga, como ha sucedido en otros países (Represa et al., 2005). Por otra parte, los sectores de la demanda que más diversidad de actividades generaron con las OVTT fueron los relacionados con los de servicios y de la construcción. En Argentina, en los últimos años este sector ha crecido considerablemente, y se ha convertido en uno de los más importantes generadores de empleo. Al analizar la variable cantidad se verifica que la actividad de asesoramiento generó el mayor número de acuerdos contractuales que se gestionan en las OVTT privadas y públicas e involucró a todos los sectores de la demanda analizados. Los sectores primario e industrial fueron lo que ejecutaron el mayor número de contratos de I+D.

Debido a su complejidad, y a que excede el propósito de este trabajo, no se analizará en profundidad el factor geográfico. Sin embargo, vale destacar que las OVTT de la Región Centro son las que gestionan la mayor cantidad y diversidad de contratos con los sectores de la demanda, resultado que no debe sorprender ya que esta región, como fue señalado en las primeras secciones del trabajo, es la de mayor concentración de capacidades científicas y tecnológicas y de actividad industrial y económica de la República Argentina.

Por último, el análisis de regresión destinado a comparar las variables que influyen tanto en la diversidad como en la cantidad de mecanismos de interacción, atendiendo a las relacionadas con las características de las OVTT y con las del sector de la demanda, aportó información previsible.Así, la diversidad y cantidad de mecanismos de gestión crece a medida que aumenta la experiencia, el número de integrantes de la OVTT, la variedad de sectores de la demanda y el número de demandantes.

\section{Conclusión}

Más allá de que ya han transcurrido casi veinte años desde los inicios de estas Oficinas de Vinculación Tecnológica y Transferencia, su comportamiento y evolución han sido totalmente dispares. Es por ello que, para obtener resultados que sustenten los esfuerzos que se vienen realizando desde el sistema científico tecnológico en esta materia y que puedan persistir en el tiempo, es necesario contar con políticas activas por parte del Estado, que permitan aunar criterios, normativas, estrategias y recursos económicos para mejorar el desempeño de las OVTT, al menos las de carácter público.

En los últimos años, desde la órbita nacional, se vienen desarrollando diversas acciones acompañadas de incrementos presupuestarios que intentan fortalecer la integración de los diferentes actores del sistema nacional de innovación argentino, generando condiciones propicias para alentar una relación más afianzada entre ellos y el medio socioproductivo. En esa misión, las OVTT universitarias deberán ser destinatarias de una atención y tratamiento especial por parte del Estado. En ese sentido, las Universidades Nacionales en Argentina constituyen el eslabón fundamental para la generación de conocimientos científicos y tecnológicos a partir de la acción de grupos de investigación de prestigio a nivel internacional. Su indelegable misión es la formación de recursos humanos de excelencia que retroalimentan al propio sistema nacional de ciencia y tecnología o pasan a integrar los sistemas productivos del sector privado que son los que motorizan la industria y la economía nacional. La Argentina se encuentra en plena gestación de una nueva especialización industrial. Las mayores posibilidades de éxito avanzan sustentablemente en el camino de la competitividad, y estarían dadas si se fortalecen y privilegian a las industrias basadas en la incorporación de progreso técnico y de recursos humanos calificados, que articulen un proceso de transformación con crecimiento y mayor equidad (Kosacoff, 1993).

En lo que respecta a este artículo, los resultados obtenidos y el exhaustivo análisis de los mismos dan cuenta de las características y comportamiento de las estructuras de interfaz que gestionan la relación entre sectores científicotecnológicos y los productivos en la República Argentina. Los resultados aportan información cuantitativa que puede servir de base a las propias oficinas para realizar un análisis crítico de sus fortalezas y debilidades particulares y a los distintos organismos de ciencia y técnica, a los efectos de planificar políticas y estrategias a largo plazo para la promoción y fomento de estas indispensables estructuras de interfaz. Finalmente, un valor agregado de este trabajo lo constituye la solidez de los análisis estadísticos de los datos y la originalidad que le imprime la aplicación de un modelo que permite analizar y explicar en conjunto las complejas relaciones que se establecen entre las variables estudiadas. 


\section{Referencias}

AUDRETSCH, D.B., Feldman, M.P. (1996). R\&D Spillovers and the Geography of Innovation and Production. The American Economic Review, 86 (3), 630-640

AUDRETSCH, D.B.; Lehmann, E.E., Warning, S. (2005). University spillovers and new firm location. Research Policy, 34, I I I3-I I22. DOI: 10.10 I6/j.respol.2005.05.009

AUDRETSCH, D.B.;Aldridge,T., Oettl,A. (2006). The Knowledge Filter and Economic Growth: The Role of Scientist Entrepreneurship. Prepared for the Ewing Marion Kauffman Foundation. DOI: 10.2139/ssrn. 1456458

BRANSCOMB, L.M., Kodama, F., Florida, R., (1999). Industrializing knowledge, University-industry Linkages in Japan and the United States. The MIT press Cambridge MA.

CARLSSON, B. (1994). Technological Systems and Economic Performance. In: Dodgson, M., Rothwell, R. (Eds.). The Handbook of Industrial Innovation, Pp.13-24. Cheltenham: Edward Elgar.

CURI, C.; Daraio, C., Llerena, P. (20I2). University technology transfer: how (in)efficient are French universities? Cambridge Journal of Economics, 36, 629-654. DOI: 10.1093/cje/ bes 020

D'ESTE, P., Patel, P. (2007). University-industry linkages in the UK: What are the factors underlying the variety of interactions with industry? Research Policy, 36, 1295-1313. DOI: 10.1016/j.respol.2007.05.002

ETZKOWITZ, H., Leydesdorff, I. (2000). The dynamics of innovation: from National System and "Mode 2" to a Triple Helix of University-industry-government relations. Research Policy 29, 109-123. DOI: 10.1016/S0048-7333(99)00055-4

FERNÁNDEZ-DE-LUCIO, I.; Conesa, F.; Garea, M.; Castromartínez, E.; Gutiérrez,A., Bodegas, M.A. (I996). Estructuras de interfaz en el Sistema español de Innovación Su papel en la difusión de la tecnología. Valencia: Universidad Politécnica de Valencia.

HAIR, J.; Anderson, R.; Tatham, R., Black, W. (1998). Multivariate data analysis ( $5^{\mathrm{a}}$ Edition). Prentice Hall. New Jersey.

HOWELLS, J. (2006). Intermediation and the role of intermediaries in innovation. Research Policy, 35, 7I5-728. DOI: 10.1016/j.respol.2006.03.005

FREEMAN, C. (1987). Technology Policy and Economic Performance: Lessons from Japan. Frances Pinter, London.
GLAESER, E.; Kallal, H.d.; Scheinkman, J.A., Shleifer, A. (1992). Growth in Cities. Journal of Political Economy, 100 (6), I 126 I I52. DOI: 10.1086/26|856

KOSACOFF, B. (1993). La industria argentina: Un proceso de reestructuración desarticulada. In: Desarrollando capacidades competitivas. Estrategias empresariales, internacionalización y especialización productiva de la Argentina. Informe de la Comisión Económica para América Latina y el Caribe (CEPAL), Oficina en Buenos Aires.

LEYDESDORFF, L., Meyer, M. (2007). The Triple Helix of university-industry-government relations (Introduction to the topical issue). Scientometrics, 58, 2, I9I-203. DOI: 10.1007/ sl II 92-007-0200-y

LUNDVALL, B.A. (1992). Introduction. In: Lundvall, B.A. (Ed.), National Systems of Innovation:Towards a Theory of Innovation and Interactive Learning. Pinter, London, Pp. I-19.

MANSFIELD, E. (1995). Academic research underlying industrial innovations: sources, characteristics, and financing. The Review of Economic and Statistics, 77, 55-65. DOI: 10.2307/2109992

MOLAS-GALLART, J., Salter, A., Patel, P., Scott, A., Duran, X. (2002). Measuring Third Stream Activities. Final Report to the Russell Group of Universities. Science and Technology Policy Research (SPRU), University of Sussex. Brighton (UK).

MOLAS-GALLART, J.; Castro-Martínez, E., Fernández-de-Lucio, I.(2008).Interface Structures:knowledge transfer practice in changing environments. Ingenio Working Papers 2008/4. Available at: http://digital.csic.es/bitstream/I026I/3623/I/Interface\%20Structures.pdf

NELSON, R.R. (1993). A retrospective. In: Nelson, R.R. (Ed.), National Innovation Systems: A Comparative Analysis. Oxford University Press, New York, 505-523.

REPRESA-SÁNCHEZ, D.; Castro-martínez, E., Fernández de Lucio, I. (2005). Encouraging Protection of Public Research Results in Spain. Journal of Intellectual Property Rights 10, September, 382-388

SIEGEL, D.S.; Waldman, D., Link, A. (2003). Assessing the impact of organizational practices on the relative productivity of university technology transfer offices: an exploratory study. Research Policy, 32, 27-48. DOI: 10.1016/S00487333(01)00196-2

STIRLING, A. (1998). On the economics and analysis of diversity. SPRU Electronic Working Paper No. 28, University of Sussex. Available at: http://citeseerx.ist.psu.edu/viewdoc/do wnload?doi= | 0.1. I. I 44.8865\&rep=rep | \& type=pdf 


\section{Anexos}

\begin{tabular}{|l|lcccccc|} 
& & Media & Mediana & Desv. típ. & Mínimo & Máximo & $\begin{array}{c}\text { Núm. } \\
\text { Obs. }\end{array}$ \\
\hline \multirow{5}{*}{ OVTT } & Diversidad & 2,34 & 3 & 1,19 & 0 & 4 & 71 \\
& Cantidad & 0,61 & 0 & 0,98 & 0 & 4 & 71 \\
& Experiencia & 11,07 & 10 & 7,85 & 0 & 43 & 71 \\
& Empleados (In) & 1,81 & 1,79 & 0,89 & 0 & 4,72 & 71 \\
& Doctor & 7,13 & 0 & 13,60 & 0 & 58,33 & 71 \\
& Ingresos & 46,55 & 30 & 40,34 & 0 & 100 & 71 \\
& Tipo & 0,58 & 1 & 0,50 & 0 & 1 & 71 \\
Demandante & Tipo demandante & 2,55 & 3 & 1,05 & 0 & 4 & 71 \\
& Proximidad Geográfica & 0,72 & 1 & 0,45 & 0 & 1 & 70 \\
\hline
\end{tabular}

ANEXO I: Estadísticos descriptivos

\begin{tabular}{|lllll|} 
& I+D & Asesoramiento & Formación & Comercialización \\
\hline I+D & 1 & & & \\
Asesoramiento & $0,490^{* *}$ & 1 & & \\
Formación & $0,409^{* *}$ & $0,668^{* *}$ & 1 & \\
Comercialización & $0,284^{*}$ & $0,470^{* *}$ & $0,267^{*}$ & 1 \\
\hline
\end{tabular}

ANEXO II: Matrices de correlación

a) Correlaciones de Spearman entre los mecanismos de interacción

Notas: ** La correlación es significativa al nivel I\%.* La correlación es significativa al nivel 5\%.

\begin{tabular}{|c|c|c|c|c|c|c|c|c|c|}
\hline & Tolerancia & 1 & 2 & 3 & 4 & 5 & 6 & 7 & 8 \\
\hline 1 Experiencia & 0,720 & 1,000 & & & & & & & \\
\hline 2 Empleados (In) & 0,690 & 0,311 & 1,000 & & & & & & \\
\hline 3 Doctor & 0,683 & 0,312 & 0,465 & 1,000 & & & & & \\
\hline 4 Ingresos & 0,643 & 0,244 & $\mathbf{0 , 3 1 3}$ & 0,137 & 1,000 & & & & \\
\hline 5 Tipo & 0,688 & $-0,107$ & $-0,246$ & $-0,169$ & $-0,524$ & 1,000 & & & \\
\hline 6 Sector demandante & 0,799 & 0,191 & 0,206 & 0,002 & 0,092 & 0,123 & 1,000 & & \\
\hline 7 Tipo demandante & 0,904 & 0,075 & $-0,046$ & $-0,060$ & 0,210 & $-0,132$ & 0,098 & 1,000 & \\
\hline 8 Proximidad Geográfica & 0,834 & $-0,172$ & $-0,321$ & $-0,141$ & $-0,279$ & 0,098 & $-0,075$ & $-0,054$ & 1,000 \\
\hline
\end{tabular}

b) Correlaciones para las variables independientes. Nota: En negrita las correlaciones significativas. 


\section{Notas}

'Según Howells (2006), los intermediarios también

pueden ser considerados como procesos o actividades.

${ }^{2}$ Entre las debilidades citadas por algunos autores se encuentran: falta de competencia, capacidad tecnológica de los proveedores o inadecuadas capacidades de investigación, entre otros (Carlsson, 1994).

3"Relevamiento y Plan de Fortalecimiento de las Oficinas de Vinculación Tecnológica (OVTT)" consultoría realizada por Universidad Nacional de Mar del Plata (UNMdP), Univer-sidad Nacional del Litoral (UNL), Universidad Nacional de Tres de Febrero (UNTREF), Universidad Tecnológica Na-cional (UTN), Universidad Nacional de Cuyo (UNCU), In-stituto INGENIO (España) y Consultora TOP (Argentina). Solicitada y financiada por el MINCyT a través de préstamo BID (20I0-20II).

${ }^{4}$ Este número fue previsto en los términos de referencia del propio informe.

${ }^{5}$ El análisis de correlaciones de Spearman (ver Anexo II a) entre las variables relacionadas con la modalidad de los contratos utilizados evidencia valores estadísticamente significativos de correlación $(P<0.01)$ entre los contratos de formación de recursos humanos y los de asesoramiento. Por el contrario, a pesar de su significación estadística $(P<0.0 \mathrm{I})$ los restantes mecanismos de interacción evidenciaron una baja correlación. Estos resultados permiten concluir que cada categoría representa un mecanismo de interacción en sí mismo.

"Los autores denominan "Variety I" y "Variety 2" y en nues-tro caso hemos utilizado los términos "Diversidad" $y$ "Canti-dad", respectivamente.

${ }^{7}$ Las frecuencias para esta variable son: el $2,8 \%$ no tienen demandantes de servicios; en el 12,7\% los demandantes de servicios pertenecen a I sólo sector; en el $32,4 \%$ a 2 sectores diferentes; en el $31 \%$ a 3 ; y en el 21 , I\%a los 4 sectores. 